\title{
Spontaneous QBO-like oscillations in an atmospheric model dynamical core
}

\author{
Weiye Yao $^{1}$ and Christiane Jablonowski ${ }^{1}$ \\ Received 26 May 2013; revised 2 July 2013; accepted 4 July 2013; published 26 July 2013.
}

[1] The ability of general circulation models (GCMs) to simulate the quasi-biennial oscillation (QBO) is an important model characteristic. Typically, the moist convective parameterization is believed to be the key GCM component that triggers tropical waves, thereby forcing wave-mean flow interactions. We show that QBO-like oscillations can also be simulated in a dry dynamical core driven by the Held-Suarez forcing. No gravity wave drag parameterization is applied. The simulations utilize the semi-Lagrangian spectral transform dynamical core of National Center for Atmospheric Research's Community Atmosphere Model. The QBO-like signal has a long period between 42-45 months and occurs in the upper stratosphere; different from observations. However, the amplitudes, asymmetries, and meridional extent closely resemble the observed QBO. Wave-number frequency analysis shows that resolved equatorially trapped waves are abundant despite the absence of cumulus convection. A Transformed Eulerian-Mean analysis suggests that the divergence of the Eliassen-Palm flux and vertical advection provide most of the forcing counteracted by diffusion. Citation: Yao, W., and C. Jablonowski (2013), Spontaneous QBOlike oscillations in an atmospheric model dynamical core, Geophys. Res. Lett., 40, 3772-3776, doi:10.1002/grl.50723.

\section{Introduction}

[2] The quasi-biennial oscillation (QBO) is a phenomenon in the equatorial stratosphere that occupies the region between 100 and $3 \mathrm{hPa}$. This region is characterized by a downward propagating zonal wind regime that periodically changes from westerlies to easterlies. The observed QBO period is about 22 to 34 months, with an average of approximately 28 months. The oscillation is primarily driven by upward propagating waves and their interaction with the mean flow [Holton and Lindzen, 1972]. In particular, trapped equatorial Kelvin and mixed-Rossby gravity (MRG) waves provide large portions of the westerly and easterly momentum forcing, respectively. In addition, inertia-gravity (IG) waves and small-scale gravity waves are other main contributors to the forcing [Baldwin et al., 2001].

[3] Although the QBO is a tropical oscillation, its impact is felt throughout the global stratosphere, and even in the troposphere and mesosphere. Therefore, the ability of an atmospheric general circulation model (GCM) to simulate

\footnotetext{
${ }^{1}$ Department of Atmospheric, Oceanic and Space Sciences, University of Michigan, Ann Arbor, Michigan, USA.

Corresponding author: W. Yao, Department of Atmospheric, Oceanic and Space Sciences, University of Michigan, 2455 Hayward St, Ann Arbor, MI 48109, USA. (weiyeyao@umich.edu)

(C)2013. American Geophysical Union. All Rights Reserved. 0094-8276/13/10.1002/grl.50723
}

the QBO is considered an important model characteristic. Progress has been made in simulating spontaneously generated QBOs in GCMs since 1996. However, the mix of waves and their wave-mean flow interaction mechanisms are rather different in each GCM. Some models exclusively drive the QBO by resolved waves, which are triggered by the moist convection parameterization [Hamilton et al., 2001]. Such simulations need to be viewed with care since overreactive convective schemes can lead to unrealistic precipitation [Takahashi, 1999]. Other models employ an additional gravity wave drag (GWD) parameterization to provide an extra momentum source that mimics the effects of unresolved small-scale gravity waves [Giorgetta et al., 2006; Xue et al., 2012]. But since various GWD schemes are prevalent in GCMs, their net momentum forcing varies widely and necessitates the empirical tuning of the GWD strength to obtain a realistic oscillation [Giorgetta et al., 2006]. It is thereby possible that GCMs exhibit QBO-like oscillations for very different, possibly wrong or error-compensating, reasons in comparison to nature. Giorgetta et al. [2006] also reported on the sensitivity of the QBO simulation to the vertical grid spacing. They found that stratospheric grid spacings of around $1 \mathrm{~km}$ or finer were required, which has been reaffirmed by most studies. Such fine grid spacings ensure that upward traveling waves and their wave-mean flow interactions are captured.

[4] All aforementioned 3-D GCM simulations of the QBO were driven by comprehensive physical parameterization packages. Such setups incorporate highly nonlinear interactions between, for example, the dynamical core and moist convection or the GWD parameterization. This makes it difficult to distinguish the causes and effects of waves and their wave damping mechanisms. This paper aims at disentangling these interactions. It focuses on the role of a dry GCM dynamical core in QBO-like simulations that omit the typical wave triggers like moist convection or GWD, which are generally believed to be paramount for the QBO wave forcing. In particular, we discuss the curious finding that spontaneous QBO-like oscillation are supported in the semi-Lagrangian (SLD) dynamical core of the Community Atmosphere Model (CAM) [Neale et al., 2010] that has been developed at the National Center for Atmospheric Research (NCAR). The dynamical core is isolated from the physical parameterizations via the Held and Suarez [1994] forcing. The study thereby allows an in-depth look at the wave characteristics of the dry dynamics in the QBO region, assesses the wave-mean flow interactions through Transform Eulerian Mean (TEM) analysis, and sheds light on the role of diffusion as a contributing, mostly counteracting, switching mechanism.

[5] The paper provides a description of the experimental design in section 2 . Section 3 presents the physical 

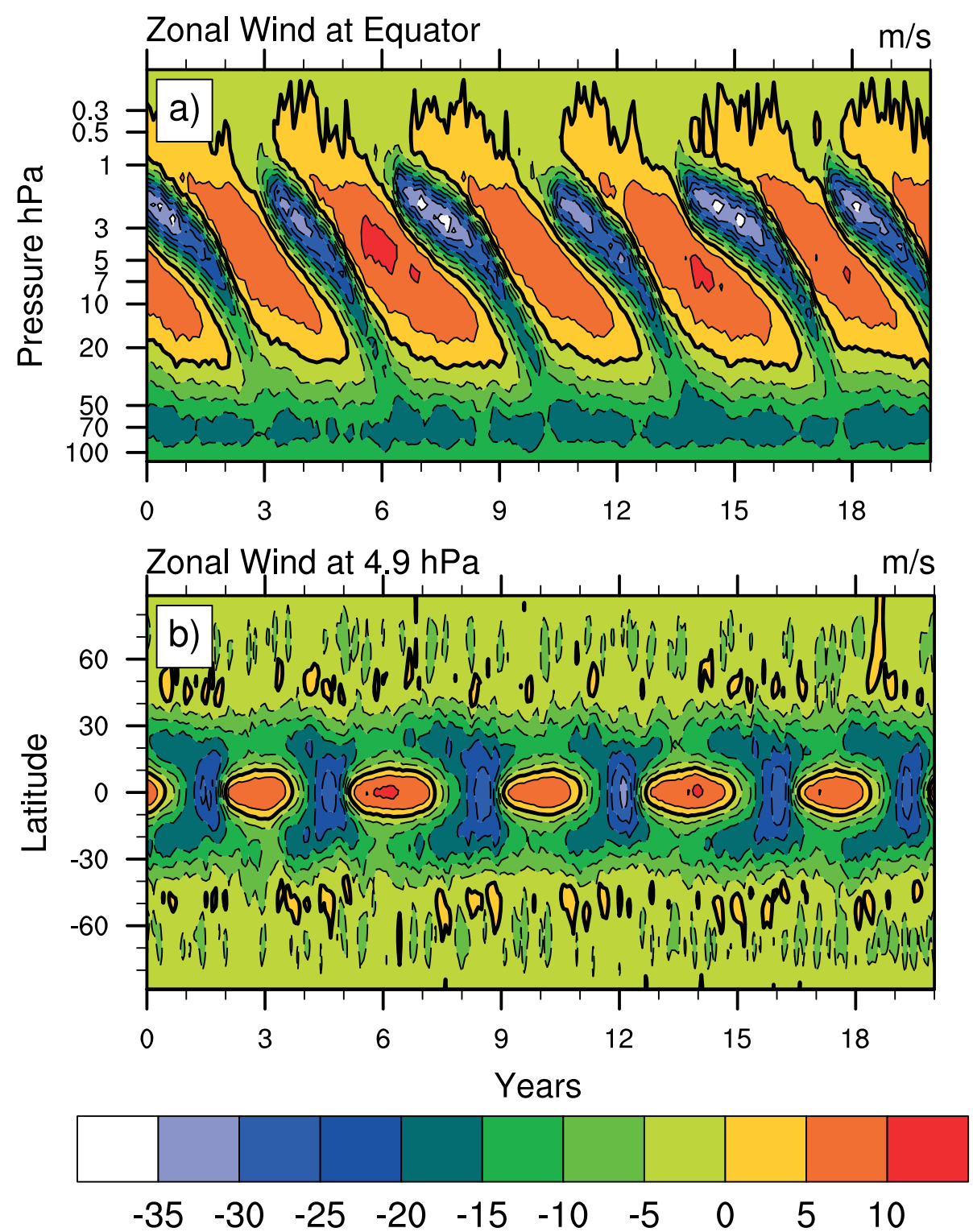

Figure 1. Monthly-mean zonal-mean zonal wind: (a) time-pressure cross section averaged between $2^{\circ} \mathrm{N} / \mathrm{S}$, (b) timelatitude cross section at $4.9 \mathrm{hPa}$.

characteristics of the QBO-like simulation, followed by wave number-frequency and TEM analyses in section 4. Conclusions are given in section 5.

\section{Experimental Design}

[6] Our study is based on an idealized 20-year long simulation with the spectral SLD dynamical core in the CAM version 5.0 (CAM5) framework. The SLD dynamical core is a two-time-level, semi-implicit, semi-Lagrangian spectraltransform model that utilizes a quadratic Gaussian transform grid. We run the model with 55 vertical levels and a model top at $0.1 \mathrm{hPa}$. The maximum vertical grid spacing is about $1.25 \mathrm{~km}$ in the stratosphere. The horizontal resolution is a triangular truncation of $\mathrm{T} 63$, which corresponds to about 1.875 degrees $(208 \mathrm{~km})$ grid spacing near the equator. The time step is $\Delta t=2700 \mathrm{~s}$. Neither a total energy fixer, nor explicitly applied diffusion is used. However, the cubic interpolations in the semi-Lagrangian scheme introduce implicit numerical diffusion which resembles fourth-order hyperdiffusion as argued by McCalpin [1988]. The model is initialized with a snapshot of a spin-up SLD run after the QBO-like oscillations have become regular.

[7] The QBO-like simulation is driven by the idealized Held and Suarez [1994] (HS) physics forcing that replaces CAM's physical parameterization package. Neither topography nor moisture are present. The Held-Suarez forcing consists of Rayleigh friction (RF) of the low-level winds and a Newtonian relaxation of the temperature field toward a prescribed equilibrium temperature which is isothermal above $100 \mathrm{hPa}$. These forcings thereby mimic the effects of the boundary-layer friction and radiation. The Newtonian damping rate in the stratosphere is $1 / 40$ day $^{-1}$. Additionally, we apply RF $\left(-K_{r} u\right)$ to the zonal wind field $u$ from $1 \mathrm{hPa}$ upward to dissipate upward traveling waves and minimize wave reflections at the model top. In essence, the additional RF acts like a sponge layer. Following Boville [1986], the expression for the pressure-dependent RF 
coefficient is given by $K_{r}=k_{0} *\left(1+\tan h\left(\left(z-z_{0}\right) / H_{0}\right)\right)$. The damping coefficient $k_{0}$ is set to $1 / 3$ day $^{-1}$, the log-pressure height is $z=h_{0} \ln \left(p_{0} / p\right), p$ symbolizes pressure, the reference pressure $p_{0}$ is set to $1000 \mathrm{hPa}$, and $h_{0}$ stands for a scale height of $7 \mathrm{~km}$. In addition, the parameter for the model top $z_{0}$ is set to $61 \mathrm{~km}$ and the scale factor $H_{0}$ is $7.7 \mathrm{~km}$. This leads to the damping time scales $1 / K_{r}$ of about 41 days at 1 $\mathrm{hPa}$ and 2 days at the model top with $0.1 \mathrm{hPa}$.

\section{Characteristics of the QBO-Like Oscillation}

[8] The SLD dynamical core spontaneously generates a QBO-like oscillation in the tropical stratosphere with periods between 42 and 45 months (43.5 months on average). This is shown in Figure 1a that displays the time-pressure cross section of the monthly-mean zonal-mean zonal wind averaged between $\pm 2^{\circ} \mathrm{N} / \mathrm{S}$. The vertical domain of the oscillation extends from $1 \mathrm{hPa}$ to about $30 \mathrm{hPa}$ in the westerly phase, and to about $50 \mathrm{hPa}$ in the easterly phase before the easterly branch transitions into the steady easterly jet between $100-50 \mathrm{hPa}$. The vertical position of the oscillation is too high in comparison to observations $(100-3 \mathrm{hPa})$ which is a common issue in many QBO simulations [Giorgetta et al., 2006; Xue et al., 2012]. The period is longer than the observed QBO period due to a weaker wave forcing in the SLD simulation, since moist convective processes or GWD are absent. The onset of the QBO-like oscillation in SLD at $1 \mathrm{hPa}$ coincides with the bottom position of the RF sponge layer, which damps the oscillation effectively between 1 and $0.1 \mathrm{hPa}$.

[9] The SLD simulation exhibits many realistic features. The westerly phases descend faster than the easterly phases and have lower amplitudes, in agreement with observations. The minimum/maximum velocity range between $-35 \mathrm{~m} \mathrm{~s}^{-1}$ and $10 \mathrm{~m} \mathrm{~s}^{-1}$ is also met rather well. The asymmetry of the oscillating wind regime is connected to the differing strengths of the driving waves. In addition, the westerly phases which last 28-32 months at $10 \mathrm{hPa}$, are longer-lived in the lower QBO domain and the time between maximum easterlies and maximum westerlies is generally shorter than the other way around.

[10] Figure $1 \mathrm{~b}$ shows the time-latitude cross section of the mean zonal wind at $4.9 \mathrm{hPa}$ which is close to the region of maximum amplitude. The figure confirms that the oscillating wind regime is a tropical phenomenon. It is confined to the tropical region between $\pm 30^{\circ}$ with a half width of about $10^{\circ}-12^{\circ}$. Note though that the westerly winds have a narrower meridional width than the observed QBO westerlies [see Pawson and Fiorino, 1998].

\section{Analysis}

[11] In order to examine the resolved wave activity, an equatorial wave number-frequency analysis is utilized following the method in Wheeler and Kiladis [1999]. The analysis (Figure 2) is based on 6-hourly instantaneous temperature data that were collected for a 50 month time period from approximately year 7 onward. The corresponding equatorial monthly-mean zonal-mean zonal wind field is displayed in Figure $3 \mathrm{a}$ by the overlaid white contours. This 50 month period is a few months longer than one complete QBO cycle. For the Wheeler-Kiladis diagrams, we choose a model level close to $22 \mathrm{hPa}$ which lies in the lower domain of
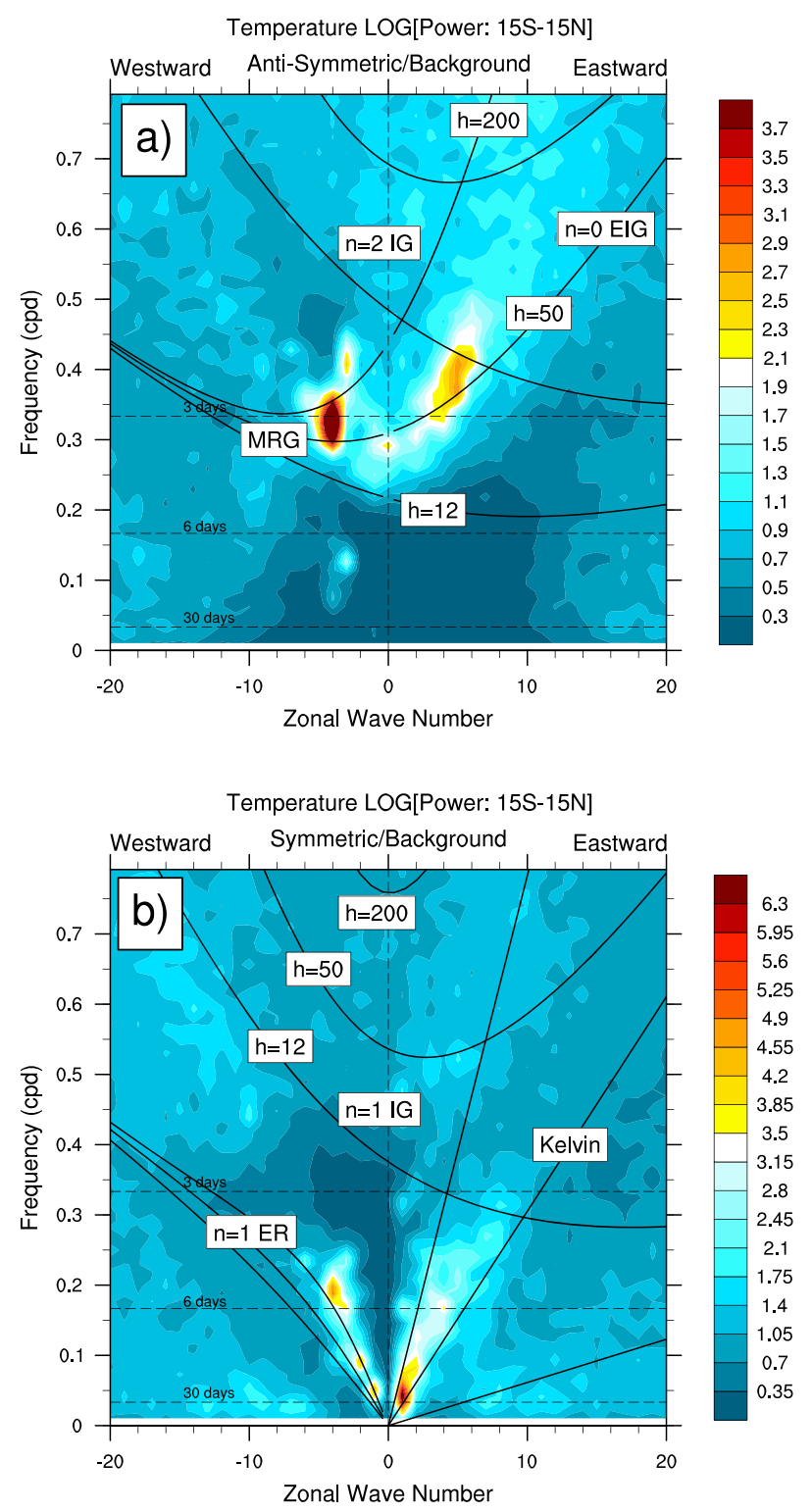

Figure 2. (a) Anti-symmetric and (b) symmetric components of the wave number-frequency spectra for the 22 $\mathrm{hPa}$ temperature, averaged between $15^{\circ} \mathrm{N} / \mathrm{S}$. The Dopplershifted dispersion curves from shallow water theory are overlaid (with $\bar{u}=-8 \mathrm{~m} \mathrm{~s}^{-1}$ ).

the QBO-like oscillation. Such a level guarantees that most of the upward traveling equatorial waves can be detected before they get filtered further upward by the ambient flow [Yang et al., 2011]. In addition, Yang et al. [2011] showed how the wave number-frequency spectra are impacted by the Doppler shift in the presence of different zonal background velocities. From linear shallow water theory, the background wind modifies the dispersion curves according to $\omega=\omega_{0}+\bar{u} k$ where $\omega$ and $\omega_{0}$ are the wave frequencies with and without the Doppler shift, $\bar{u}$ denotes the zonal background wind and $k$ stands for the zonal wave number. Figure 3 a displays that most of the region below $22 \mathrm{hPa}$ is dominated by easterlies. We therefore select $\bar{u}=-8 \mathrm{~m} \mathrm{~s}^{-1}$.

[12] In Figure 2, we overlay the Doppler-shifted dispersion curves for the equivalent depths $h$ of 12,50 , and $200 \mathrm{~m}$. 

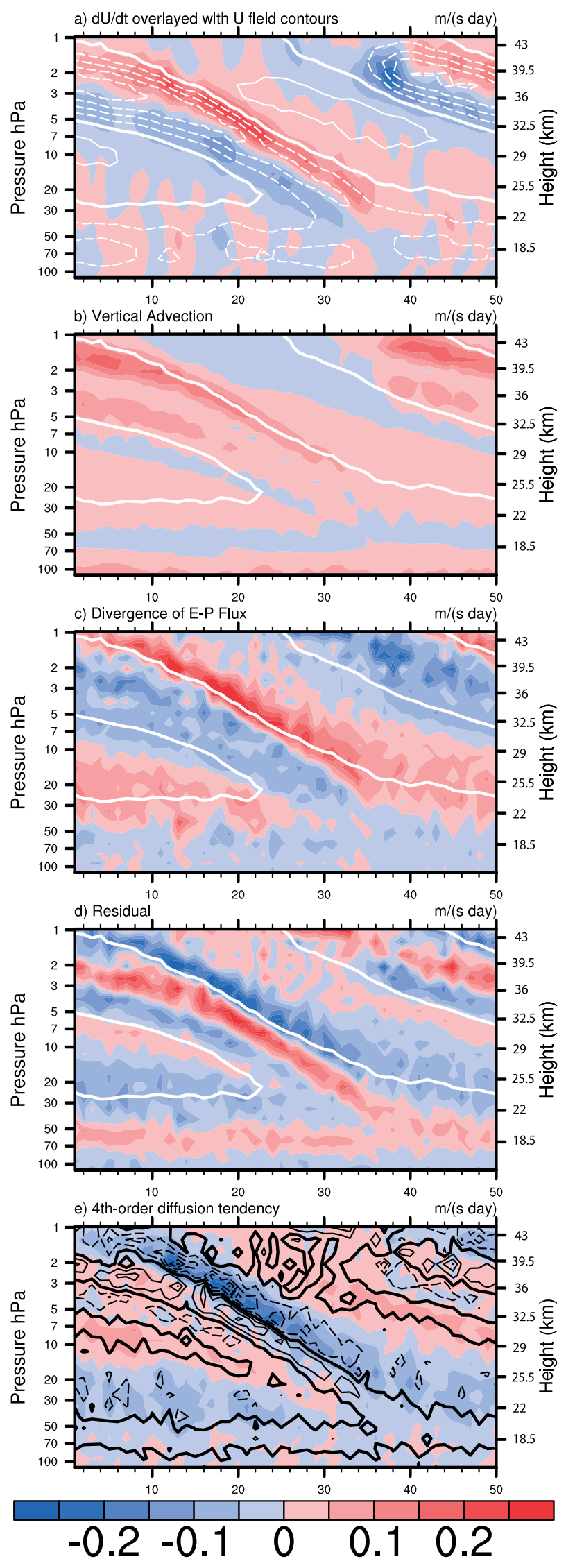
tours show the zonal-mean monthly-mean zonal wind with
Figure 3. Zonal-mean monthly-mean time-height cross sections averaged between $2^{\circ} \mathrm{N} / \mathrm{S}$ : (a) zonal wind acceleration, (b) TEM vertical advection, (c) divergence of the E-P flux, (d) TEM residual, and (e) acceleration effects of fourthorder horizontal diffusion with $K_{4}=5 \times 10^{15} \mathrm{~m}^{4} \mathrm{~s}^{-1}$. In Figure $3 \mathrm{e}$, the contours of the residual are overlaid in black with contour spacing $0.1 \mathrm{~m} \mathrm{~s}^{-1}$ day $^{-1}$. Overlaid white concontour interval $8 \mathrm{~m} \mathrm{~s}^{-1}$ in Figure 3a, only the enhanced zero wind line is shown in Figures $3 b-3 e$. All negative contours are dashed.

These equivalent depths correspond to the zonal wave speeds of about 11,22 , and $44 \mathrm{~m} \mathrm{~s}^{-1}$ and approximate vertical wavelengths $L_{z}$ of $2.8,6.2$, and $12.6 \mathrm{~km}$, assessed with the squared stratospheric Brunt-Väisälä frequency $N^{2} \approx$ $5 \times 10^{-4} \mathrm{~s}^{-2}$. This range of phase speeds and vertical wave rially trapped waves in the stratosphere like Kevin and MRG waves. Thereby, our analysis sheds light on their presence in idealized simulations that omit important wave generation metric wave number-frequency spectra which are averaged between $15^{\circ} \mathrm{N} / \mathrm{S}$. The estimated background spectrum has been removed [see Wheeler and Kiladis, 1999]. The antisymmetric plot (Figure 2a) shows a spectral peak for MRG waves with periods around 3-4 days and negative wave numbers -4 to -6 . Such negative wave numbers denote a westward propagation. The wave number-frequency range between -3 and -5 with frequencies $\leq 0.3$ cycles per day
(cpd) is also indicative of free Rossby waves. Moreover, spectral peaks are detected for eastward traveling Equatorial Inertia-Gravity (EIG) waves with meridional mode number $n=0$. These EIG waves have shorter periods of around 2-3 days within the wave number range $2-7$. At higher wave numbers, a mix of EIG and IG waves is detected. The symmetric plot (Figure 2b) displays the Doppler-shifted dispersion curves for Kelvin, Equatorial Rossby (ER), and $n=1$ IG waves. Spectral peaks for ER waves with wave
numbers -2 to -4 and periods between 5 and 20 days are apparent, as well as Kelvin waves with wave numbers 1-6 and periods between 5 and 30 days. The most dominant peaks in the Kelvin wave regime lie around wave numbers $1-2$ with 15-30 day periods. They are dominant features in the westerly momentum forcing for the QBO. This shows that equatorial stratospheric waves are abundant in the idealized simulation, even without simulating the latent heat release due to tropical cumulus convection or parameterized GWD. Therefore, the waves in SLD must have been trigpropagating normal modes.

[13] A TEM analysis is performed to investigate the wave-mean flow interaction. It is applied along constant pressure levels in the stratosphere following the pressure-
based TEM formulation in Andrews et al. [1983]. TEM identifies four drivers for the zonal-mean zonal wind acceleration; the mean meridional advection, mean vertical advection, the divergence of the Eliassen-Palm (E-P) flux which denotes the wave-mean flow interaction and the
residual. The latter incorporates diffusion and other damping mechanisms like the effects of the radiative damping. 
Figure 3 shows the 50 month time series of the monthlymean zonal-mean (Figure 3a) zonal wind acceleration, (Figures 3b-3d) TEM partitioning and (Figure 3e) an assessment of diffusion in a time-height cross section averaged between $2^{\circ} \mathrm{N} / \mathrm{S}$. The mean meridional advection is not shown because of its very small magnitudes that range between $\pm 0.03 \mathrm{~m} \mathrm{~s}^{-1}$ day $^{-1}$. The mean vertical advection (Figure $3 \mathrm{~b}$ ) mostly provides a westerly acceleration during the easterly phase. This forcing contributes to the descent of the upper (easterly-to-westerly) zero wind line where the wind acceleration (Figure $3 \mathrm{a}$ ) is positive, but slows the descent of the lower (westerly-to-easterly) zero wind line where the acceleration is negative. The vertical advection mostly counteracts the effects of the E-P flux divergence (the resolved wave forcing), which was also found by Hamilton et al. [2001], Giorgetta et al. [2006], and Xue et al. [2012]. The E-P flux divergence (Figure 3c) is the most important driver of the QBO-like oscillation which provides most of the easterly acceleration during the easterly phase and most of the westerly force during westerly phase. However, its vertical position in comparison to the wind acceleration (Figure 3a) is shifted upward as it was also displayed in Hamilton et al. [2001]. The momentum budget in the idealized simulation can thereby only be closed in the presence of a large residual (Figure $3 \mathrm{~d}$ ) that mostly plays a counteracting role in comparison to the E-P flux divergence.

[14] Figure $3 \mathrm{e}$ compares the residual to the shape of fourth-order horizontal hyperdiffusion $\left(-K_{4} \nabla^{4} \mathbf{v}\right)$ at the equator with the horizontal velocity vector $\mathbf{v}$ and the hypothetical diffusion coefficient $K_{4}=5 \times 10^{15} \mathrm{~m}^{4} \mathrm{~s}^{-1}$. This coefficient corresponds to a damping time scale of $5.6 \mathrm{~h}$ for the maximum wave number, which is a reasonable choice at the T63 resolution [Jablonowski and Williamson, 2011]. The figure suggests that the implicit numerical diffusion in SLD, which mimics fourth-order hyperdiffusion, seems to capture most of the gross tendencies of the residual. A perfect match is not expected since the nonlinear aspects of the implicit diffusion are not represented, and neither the effects of the radiative HS damping (indirectly via the thermal wind relationship) nor vertical diffusion are accounted for in this linear assessment of the diffusive tendencies. The diffusion mostly counteracts the resolved wave forcing and thereby plays a considerable role in this simulation. In general, QBO simulations in GCMs have been found to be very sensitive to their diffusive properties. Takahashi [1999] and Hamilton et al. [2001] reported that they needed to lower the coefficients of the explicitly applied fourth-order horizontal hyperdiffusion. This raises the question what the relative roles of mechanical (diffusive) versus thermal damping are in both the observed and modeled QBO, and whether the idealized SLD simulation reveals a physical or numerical effect.

\section{Conclusions}

[15] A QBO-like oscillation has been simulated in the dry CAM SLD dynamical core that was driven by the Held-Suarez forcing with additional Rayleigh friction near the model top. The QBO-like signal has an average period of 43.5 months and occupies the equatorial domain between 50 and $1 \mathrm{hPa}$. The overall shape of the oscillation resembles observations, although the QBO-like signal is located higher up in the stratosphere and its period is longer than observed. The wave-number frequency analysis showed that Doppler-shifted resolved equatorially trapped Kelvin, MRG, ER, and EIR waves are abundant in the idealized simulation despite the absence of the typical wave triggering mechanisms like cumulus convection. These waves are known to be important wave drivers for the QBO. In addition, no GWD parameterization was applied. The TEM analysis showed that the divergence of the E-P flux and vertical advection are the most important drivers of the QBO-like oscillation. However, the residual is of the same order of magnitude and mostly counteracts the resolved wave forcing. It contains the effects of dissipative processes. We found that the shape of the residual closely resembles the effects of momentum diffusion in the form of a fourth-order horizontal hyperdiffusion. This suggests that the implicit numerical diffusion from the semi-Lagrangian scheme contributes considerably to the QBO-like oscillation in the idealized simulation which might shed new light on the role of diffusion in the QBO forcing.

[16] Acknowledgments. The work was supported by the Office of Science, U.S. Department of Energy, award DE-SC0006684.

[17] The Editor thanks two anonymous reviewers for their assistance in evaluating this paper.

\section{References}

Andrews, D. G., J. D. Mahlman, and R. W. Sinclair (1983), EliassenPalm diagnostics of wave-mean flow interaction in the GFDL "SKYHI" general circulation model, J. Atmos. Sci., 40, 2768-2784.

Baldwin, M. P., et al. (2001), The quasi-biennial oscillation, Rev. Geophysics, 39(2), 179-229.

Boville, B. A. (1986), Wave-mean flow interactions in a general circulation model of the troposphere and stratosphere, J. Atmos. Sci., 43, 1711-1725.

Giorgetta, M. A., E. Manzini, E. Roeckner, M. Esch, and L. Bengtsson (2006), Climatology and forcing of the quasi-biennial oscillation in the MAECHAM5 model, J. Climate, 19, 3882-3901.

Hamilton, K., R. J. Wilson, and R. S. Hemler (2001), Spontaneous stratospheric QBO-like oscillations simulated by the GFDL SKYHI general circulation model, J. Atmos. Sci., 58, 3271-3292.

Held, I. M., and M. J. Suarez (1994), A proposal for the intercomparison of the dynamical cores of atmospheric general circulation models, Bull. Amer. Meteor. Soc., 75(10), 1825-1830.

Holton, J. R., and R. S. Lindzen (1972), An updated theory for the quasibiennial cycle of the tropical stratosphere, J. Atmos. Sci., 29, 1076-1080.

Pawson, S., and M. Fiorino (1998), A comparison of reanalyses in the tropical stratosphere. Part 2: The quasi-biennial oscillation, Clim. Dynam., 14(9), 645-658.

Jablonowski, C., and D. L. Williamson (2011), The pros and cons of diffusion, filters, and fixers in atmospheric general circulation models, in Numerical Techniques for Global Atmospheric Models, Lecture Notes in Science and Engineering, vol. 80, edited by P. H. Lauritzen et al., pp. 381-493, Springer, Berlin, Heidelberg.

McCalpin, J. D. (1988), A quantitative analysis of the dissipation inherent in semi-Lagrangian advection, Mon. Wea. Rev., 116, 2330-2336.

Neale, R. B., et al. (2010), Description of the NCAR Community Atmosphere Model (CAM 5.0), NCAR Tech. Note NCAR/TN-486+ STR, 282 pp., National Center for Atmospheric Research, Boulder, Colorado.

Takahashi, M. (1999), Simulation of the quasi-biennial oscillation in a general circulation model, Geophys. Res. Lett., 26(9), 1307-1310.

Wheeler, M., and G. N. Kiladis (1999), Convectively coupled equatorial waves: Analysis of clouds and temperature in the wavenumber-frequency domain, J. Atmos. Sci., 56, 374-399.

Xue, X., H. Liu, and X. Dou (2012), Parameterization of the inertial gravity waves and generation of the quasi-biennial oscillation, J. Geophys. Res., 117, D06103, doi:10.1029/2011JD016778

Yang, G. Y., B. J. Hoskins, and J. M. Slingo (2011), Equatorial waves in opposite QBO phases, J. Atmos. Sci., 68, 839-862. 